\title{
Observations of Piezoresistivity for Polysilicon in Bending that are Unexplained by Linear Models
}

Tyler L. Waterfall

Gary K. Johns

garykjohns@gmail.com

Robert K. Messenger

Brian D. Jensen

Timothy W. McLain

See next page for additional authors

Follow this and additional works at: https://scholarsarchive.byu.edu/facpub

Part of the Mechanical Engineering Commons

\section{Original Publication Citation}

Waterfall, T.L., Johns, G.K., Messenger, R.K., Jensen, B.D., McLain, T.W., and Howell, L.L., â€œObservations of Piezoresistivity for Polysilicon in Bending that are Unexplained by Linear Models,â€ Sensors \& Actuators A: Physical, Vol. 141, pp. 61-618, 28.

\section{BYU ScholarsArchive Citation}

Waterfall, Tyler L.; Johns, Gary K.; Messenger, Robert K.; Jensen, Brian D.; McLain, Timothy W.; and Howell, Larry L., "Observations of Piezoresistivity for Polysilicon in Bending that are Unexplained by Linear Models" (2008). Faculty Publications. 882.

https://scholarsarchive.byu.edu/facpub/882

This Peer-Reviewed Article is brought to you for free and open access by BYU ScholarsArchive. It has been accepted for inclusion in Faculty Publications by an authorized administrator of BYU ScholarsArchive. For more information, please contact ellen_amatangelo@byu.edu. 


\section{Authors}

Tyler L. Waterfall, Gary K. Johns, Robert K. Messenger, Brian D. Jensen, Timothy W. McLain, and Larry L. Howell 


\title{
Observations of Piezoresistivity for Polysilicon in Bending that are Unexplained by Linear Models
}

\author{
Tyler L. Waterfall, Gary K. Johns, Robert K. Messenger, \\ Brian D. Jensen *, Timothy W. McLain, and Larry L. Howell \\ Department of Mechanical Engineering \\ Brigham Young University, Provo, UT 84602
}

\begin{abstract}
Compliant piezoresistive MEMS sensors exhibit great promise for improved on-chip sensing. As compliant sensors may experience complex loads, their design and implementation require a greater understanding of the piezoresistive effect of polysilicon in bending and combined loads. This paper presents experimental results showing the piezoresistive effect for these complex loads. Several $n$-type polysilicon test structures, fabricated in MUMPs and SUMMiT processes, were tested. Results show that, while tensile stresses cause a linear decrease in resistance, bending stresses induce a nonlinear rise in resistance, contrary to the effect predicted by linear models. In addition, tensile, compressive, and bending loads combine in their effects on resistance. The experimental data illustrate the inability of linear piezoresistance models to predict the piezoresistive trends of polysilicon in bending and combined loads, indicating the need for more complete nonlinear models appropriate for these loading conditions.
\end{abstract}

\section{Introduction}

Since Smith documented the piezoresistive effect in silicon in 1954 [1], piezoresistive MEMS devices have been implemented in a variety of sensing applications including pressure, acceleration, force, and displacement sensing [2-8]. Piezoresistivity - the change in electrical resistance due to applied stress or strain - facilitates the measurement of stress or strain in a silicon member. This enables the use of piezoresistive devices in on-chip and feedback-control applications $[9,10]$.

* email:bdjensen@byu.edu 


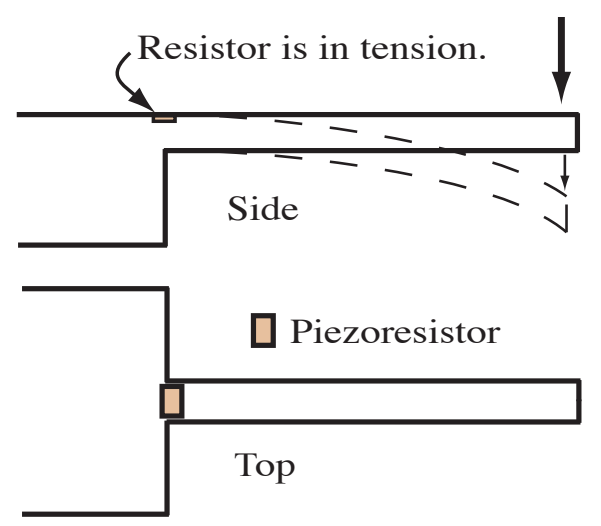

(a)

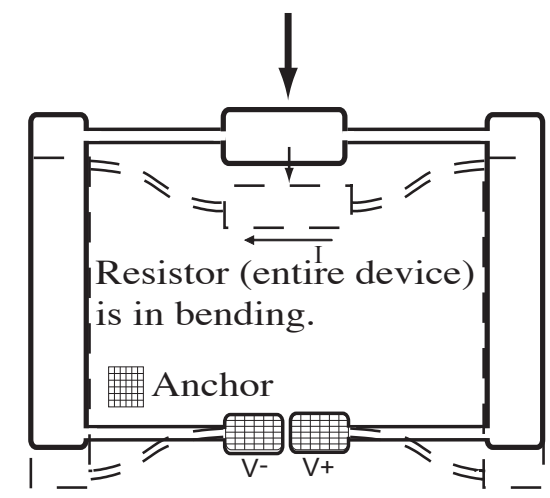

(b)

Fig. 1. (a) Traditional cantilever-beam piezoresistive force sensor and (b) integral piezoresistive force sensor.

The literature today contains extensive data, models, and theories describing the piezoresistive effect in tension and compression for both monocrystalline and polycrystalline silicon [11-20]. For most existing sensors, these data and models have been sufficient. For example, a traditional cantilever-beam force sensor, depicted in Fig. 1a, is composed of two basic components. First, a cantilever beam structure experiences the physical phenomenon, such as an applied force. Second, a piezoresistive element diffused on top of the cantilever is thin enough that it experiences nearly purely tensile or purely compressive stress as the cantilever beam deflects [21]. In this configuration, the structure and the sensing element are two distinct objects, with separate fabrication steps. Because the piezoresistive element experiences pure compression or tension during loading, traditional linear models are sufficient to model this behavior.

In contrast, Fig. 1b shows how a compliant u-shaped spring, or U-Spring device, can function as an integral piezoresistive force or displacement sensor. Such a device is fabricated from a single, uniformly-doped layer of polysilicon and allows piezoresistive sensing of the position or force of a MEMS actuator. It represents an elegant way to sense such behavior, because the sensor 
is fabricated from the same layer and mask used to create the actuator, and it simultaneously acts as both a sensor and a support spring to guide the desired motion. During motion, the spring compresses or stretches. The resultant change in resistance of the spring is measured and used to calculate the applied force or displacement. Thus, the entire structure is the piezoresistive element, and it experiences bending (rather than pure tension or compression) during motion. An understanding of the piezoresistive effect of polysilicon in bending and combined loads is vital to the design and implementation of this and other innovative piezoresistive sensors. For example, the compliant bistable mechanism with integral piezoresistive state sensing, described in [22], experiences combined bending and compressive loads during motion. A more complete nonlinear model would enhance the design of this and similar devices.

The purpose of this paper is to present the testing of several structures used to explore the piezoresistive property of polysilicon in bending and combined loads. The data are compared to the predictions of a simplified model. The model uses traditional linear representations for piezoresistance derived from Smith's $\pi$-coefficient model. The comparisons show that linear models are inadequate to predict piezoresistive behavior of polysilicon under bending loads. The need for a more complete model of piezoresistance, which accounts for the effect of bending and combined loads on resistance, is thus shown.

\section{Background}

Although the physical mechanism of piezoresistivity is not completely understood, several important trends of piezoresistance in tension and compression have been documented. For example, it has been shown that the resistance of an $n$-type (phosphorus-doped) polysilicon member subjected to uniaxialtensile stress decreases nearly linearly. For the same material, an applied compressive stress causes an approximately linear increase in resistance. The opposite is true in both cases for $p$-type (boron-doped) polysilicon, with the resistance increasing in tension and decreasing in compression.

Smith's model of piezoresistance for single-crystal silicon relates the fractional change in electrical resistivity, $\Delta \rho / \rho$, in each crystalline direction to the ap- 
plied stress, $\sigma$, with a matrix of piezoresistance $(\pi)$ coefficients as

$$
\frac{1}{\rho}\left[\begin{array}{c}
\Delta \rho_{1} \\
\Delta \rho_{2} \\
\Delta \rho_{3} \\
\Delta \rho_{4} \\
\Delta \rho_{5} \\
\Delta \rho_{6}
\end{array}\right]=\left[\begin{array}{cccccc}
\pi_{11} & \pi_{12} & \pi_{12} & 0 & 0 & 0 \\
\pi_{12} & \pi_{11} & \pi_{12} & 0 & 0 & 0 \\
\pi_{12} & \pi_{12} & \pi_{11} & 0 & 0 & 0 \\
0 & 0 & 0 & \pi_{44} & 0 & 0 \\
0 & 0 & 0 & 0 & \pi_{44} & 0 \\
0 & 0 & 0 & 0 & 0 & \pi_{44}
\end{array}\right]\left[\begin{array}{c}
\sigma_{1} \\
\sigma_{2} \\
\sigma_{3} \\
\tau_{1} \\
\tau_{2} \\
\tau_{3}
\end{array}\right]
$$

where the $\pi$ coefficients are determined experimentally $[1,12,23,24]$. Note that this model is based on observed behavior and not on fundamental physics. For polysilicon structures, which have grains arranged in many different crystal orientations, Smith's single-crystal model is often simplified to give $[25,26]$

$$
\frac{\Delta \rho}{\rho}=\pi_{l} \sigma_{l}+\pi_{t} \sigma_{t}
$$

where $\pi_{l}$ is the piezoresistance coefficient along the length of a resistor, $\pi_{t}$ is the piezoresistance coefficient transverse to the length of a resistor, and $\sigma_{l}$ and $\sigma_{t}$ are the stresses directed along the length of the resistor and transverse to the length of the resistor, respectively. Because beams in tension, compression, or bending experience a much greater stress in the direction along the length of the beam than the stresses in the other two orthogonal directions, we may assume that these lower stresses are insignificant. Therefore, equation (2) simplifies to

$$
\frac{\Delta \rho_{1}}{\rho}=\pi_{l} \sigma_{l}
$$

This paper reports research and testing that has been performed to determine the adequacy of this linear model in bending applications. It describes the collection and evaluation of data on the piezoresistive effect in bending and combined loads. Several test structures are presented and their corresponding piezoresistive behavior is compared to that predicted by linear piezoresistance models. 


\section{Test Devices and Experimental Setup}

Several test structures were designed and tested to explore the piezoresistive effects of tension, bending, and combined loads. Devices were fabricated using the MUMPs [27] and SUMMiT [28] processes which use $n$-type polysilicon as the fabrication material. For both processes, the polysilicon resistivity is between $1.5 \times 10^{-2}$ and $3 \times 10^{-2} \Omega \cdot \mathrm{cm}$. The test devices are summarized in Table 1. As shown in Fig. 2, diverse structures were designed in an attempt to capture the probable loading conditions an integral piezoresistive sensor would experience. Scanning electron micrographs of the structures are shown in Fig. 3. Dimensions and measured resistance for each design are included in Tables 2 and 3. Device names such as L150p1p2 represent a tensile structure with length $150 \mu \mathrm{m}$ and made of poly 1 (p1) and poly 2 (p2) structural layers.

First, the tension structures were tested and compared to the linear piezoresistance model to characterize any differences in piezoresistive behavior due to Table 1

Piezoresistance test structures.

\begin{tabular}{|c|c|c|}
\hline Device & Fabrication Process & Desired Testing Information \\
\hline Tension Bars & MUMPs/SUMMiT & $\begin{array}{l}\text { Tensile Model Validation; In- } \\
\text { fluence of Fabrication Method, } \\
\text { Polysilicon Layer and Beam } \\
\text { Length }\end{array}$ \\
\hline Folded-Beam & MUMPs & Effects of Bending Loads \\
\hline U-Spring & SUMMiT & Effects of Bending Loads \\
\hline S-Curl & MUMPs & Combined Loads \\
\hline Snake & MUMPs & Combined Loads \\
\hline
\end{tabular}

Table 2

Nominal dimensions and measured resistance of piezoresistive tensile and bending structures, given in $\mu \mathrm{m}$ and $\Omega$.

\begin{tabular}{lccccc} 
Device Name & Process & Length, $L_{x}$ & Width, $w$ & Thickness, $t$ & Resistance \\
\hline L100 & SUMMiT & 100 & 1 & 2.25 & 862 \\
L150p1p2 & MUMPs & 150 & 3 & 3.5 & 1,432 \\
L50p1 & MUMPs & 50 & 3 & 2 & 286 \\
L50p2 & MUMPs & 50 & 3 & 1.5 & 1,274 \\
L50p1p2 & MUMPs & 50 & 3 & 3.5 & 549 \\
Folded-Beam & MUMPs & 150 & 3 & 3.5 & 1,649 \\
U-Spring & SUMMiT & 90 & 2.75 & 4.5 & 336 \\
\hline
\end{tabular}




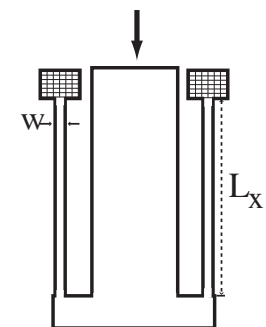

(a) Tension Bars

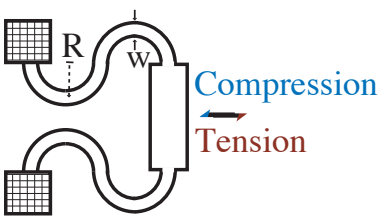

(b) S-Curl

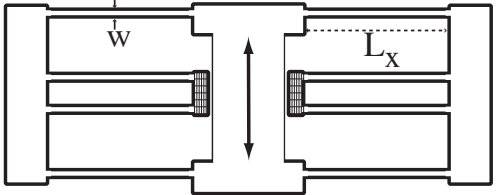

(c) Folded-Beam

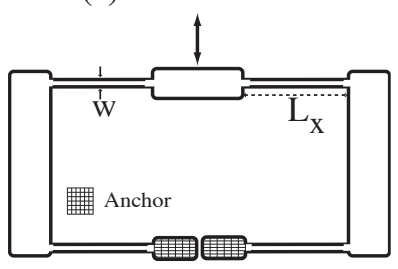

(d) U-Spring

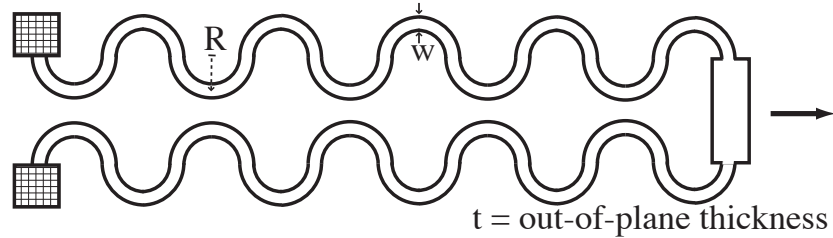

(e) Snake

Fig. 2. Test structures fabricated for the characterization of the piezoresistive effect of silicon.
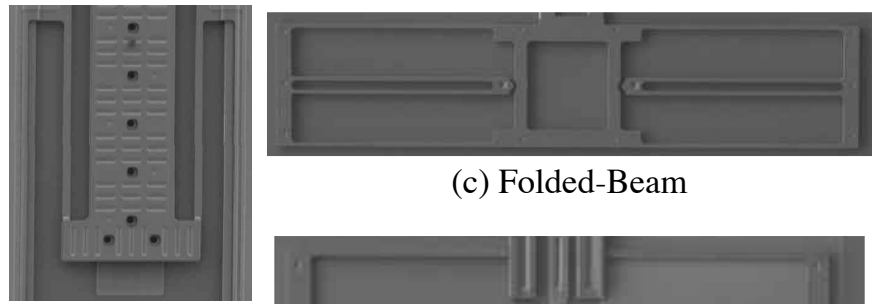

(c) Folded-Beam

(a) Tension Bars

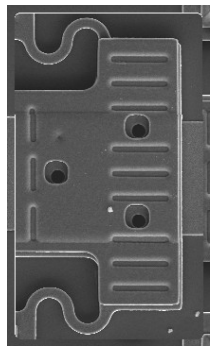

(b) S-Curl

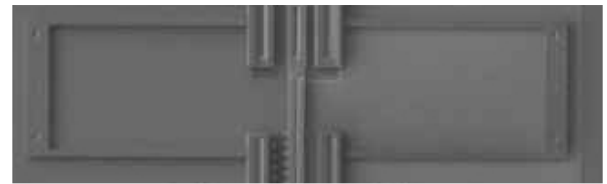

(d) U-Spring

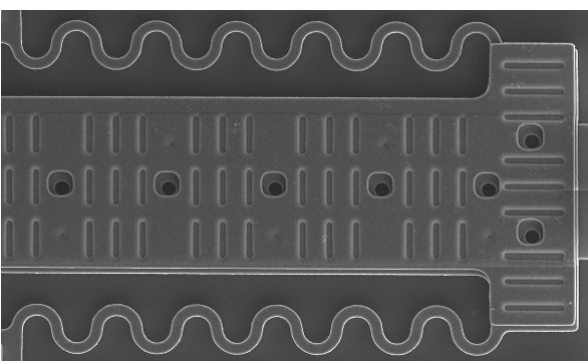

(e) Snake

Fig. 3. SEM images of test structures fabricated for the characterization of the piezoresistive effect of silicon. 
Table 3

Dimensions and measured resistance of MUMPs-fabricated piezoresistance combined load structures, given in $\mu \mathrm{m}$ and $\Omega$.

\begin{tabular}{lcccc} 
Device & Centerline Radius, $R$ & Width, $w$ & Thickness, $t$ & Resistance \\
\hline S-Curl & 4.5 & 3 & 2 & 265 \\
Snake & 4.5 & 3 & 2 & 1,149 \\
\hline
\end{tabular}

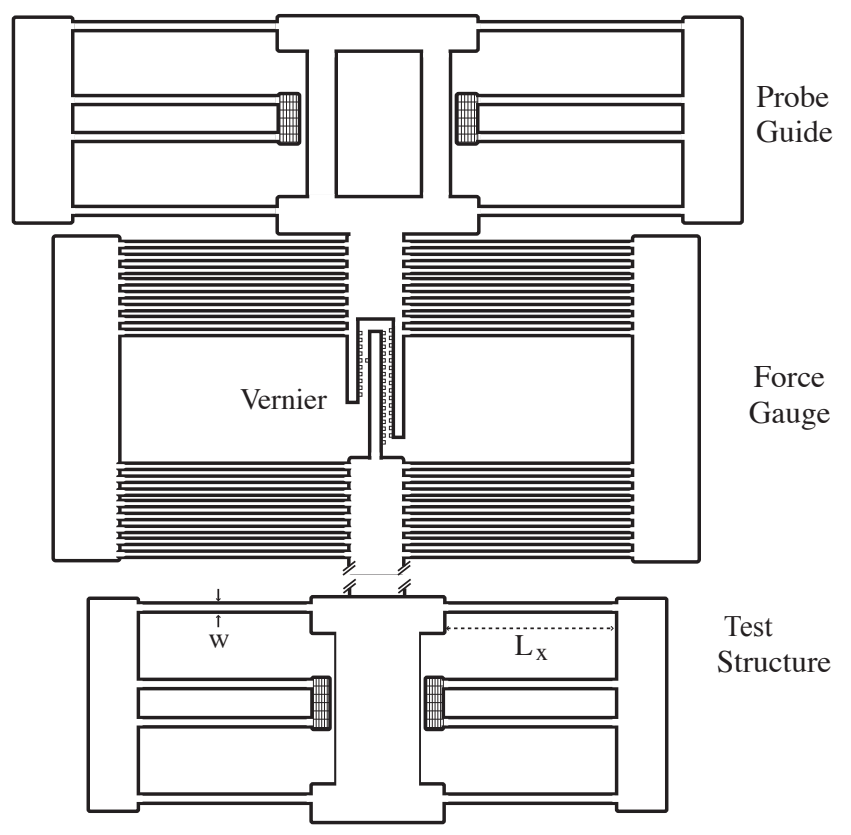

Fig. 4. Each test structure included a micro probe guide (top) to reduce off-axis forces and deflections and a linear force gauge (bottom) to allow force measurement.

the fabrication process, fabrication layers, and tensile member length. Next, piezoresistivity in bending was explored using the Folded-Beam and the USpring devices shown in Figs. 2c and 2d. Finally, piezoresistance in combined loads (bending and tension or compression) was investigated with the S-Curl and Snake structures.

Each test structure was fabricated with an attached micro-probe guide, force gauge [29], and optical vernier, as shown in Fig. 4. These structures allowed for force application and measurement. The probe guide provided off-axis stability for the microprobe used to apply the desired forces to the test structure. With its relatively low stiffness $(k=9.2 \mu \mathrm{N} / \mu \mathrm{m})$ it provided little resistance to motion in the test direction. The MUMPs-fabricated probe guide was designed with the same geometry and dimensions as the folded-beam structure depicted in Fig. 2 (refer to Table 2 for dimensions).

Forces were calculated using large deflection equations [30] for the relative deflection of the folded-beam force gauge in Fig. 4. For the MUMPs-fabricated devices, with the exception of the folded-beam device, the force gauge had a 
linear spring constant of approximately $k=147 \mu \mathrm{N} / \mu \mathrm{m}$. Because of the smaller stiffness of the folded-beam device, its force gauge was designed to have a much lower stiffness of $9.1 \mu \mathrm{N} / \mu \mathrm{m}$. A similar force gauge was attached to the SUMMiT-fabricated devices.

The amount the force gauge was compressed or stretched was measured with an optical vernier. Each row of vernier 'teeth' was offset from the facing row in such a way that the alignment of each sequential set of teeth represented a displacement of $0.5 \mu \mathrm{m}$. With this displacement resolution, a resolution of applied force of approximately $73.5 \mu \mathrm{N}$ was obtained for most of the MUMPs devices, and a force resolution of $4.6 \mu \mathrm{N}$ for the folded-beam device. The force resolution for the SUMMiT-fabricated U-Spring was $21.1 \mu \mathrm{N}$.

Once fabricated, mechanically released, and wire-bonded, the piezoresistive behavior of each device was measured using a three step process. First, the nominal electrical resistance was measured. Second, a set of specific force steps were applied, with the resistance being recorded at each step. Third, the

undeflected, or nominal, resistance was measured again in order to observe any thermal drift or plastic deformation. Most devices were tested with a progressive sweep of applied forces.

\section{Experimental Results}

The following subsections present the experimental results for each test device and provide a summary of the significance of each data set, including tensile, bending, and combined loading conditions.

\subsection{Tensile Loads}

The goals of tensile testing were to observe the variation in piezoresistive behavior due to the fabrication process and to compare test results to the linear piezoresistive model. The results of both SUMMiT- and MUMPs-fabricated tensile elements of various geometries illustrate how the electrical resistance decreased linearly as the applied tensile stress increased, as shown in Fig. 5. This piezoresistive behavior in tension followed the linear trend described in the literature for $n$-type polysilicon $[1,15]$.

For the SUMMiT tensile device, the piezoresistive sensitivity (as approximated by the slope of the linear region) is $0.011 \%$ decrease in resistance per MPa. A similar linear decrease in resistance was evident in the MUMPs-fabricated devices, with a sensitivity ranging from $0.010-0.012 \%$ change in resistance per 


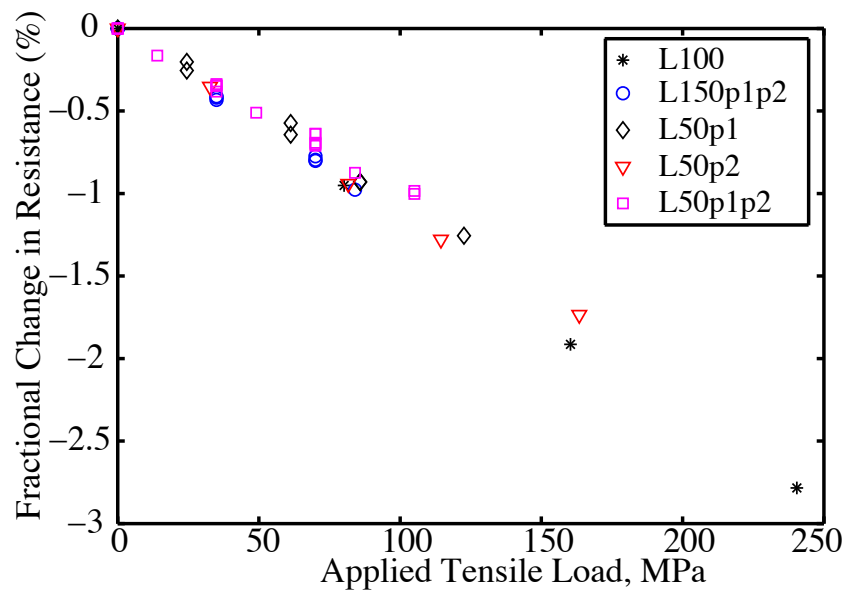

Fig. 5. Comparison of piezoresistance in tensile devices for MUMPs- and SUMMiTfabricated devices of varying length and fabrication layer.

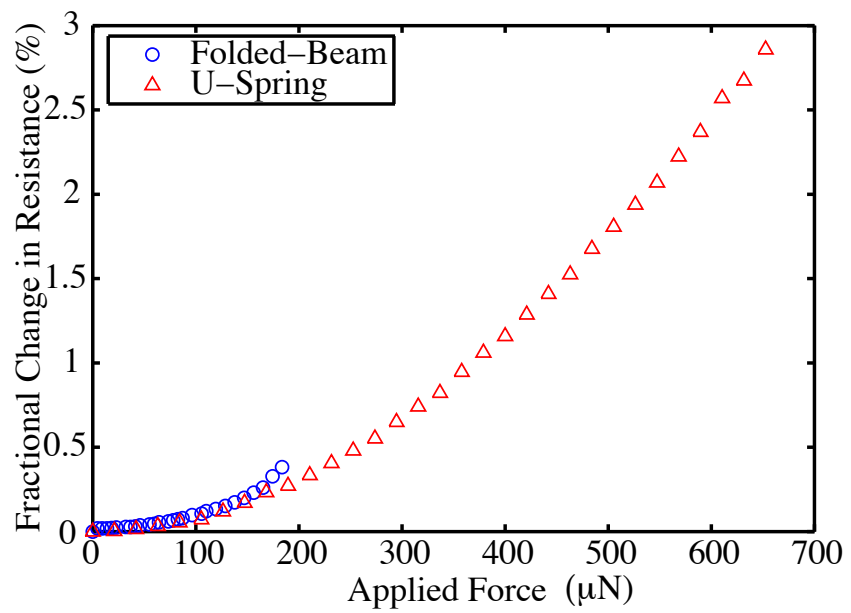

Fig. 6. Piezoresistance behavior of SUMMiT-fabricated U-Spring device and of Folded-Beam device fabricated with the POLY1 and POLY2 layers of the MUMPs process.

MPa. The variation of piezoresistive behavior among the MUMPs tensile elements was explained by the differences in element length and polysilicon layer, with a slightly higher sensitivity (steeper slope) corresponding to longer tensile elements fabricated in POLY2 (the second structural layer deposited during MUMPs fabrication). It should be noted, however, that the measured differences in piezoresistive sensitivity among polysilicon layers was quite small.

\subsection{Bending Loads}

The U-Spring and Folded-Beam devices were designed to characterize the piezoresistance effect for bending loads. The resistance change was induced by deflection caused by an applied force, much like the tensile devices. 


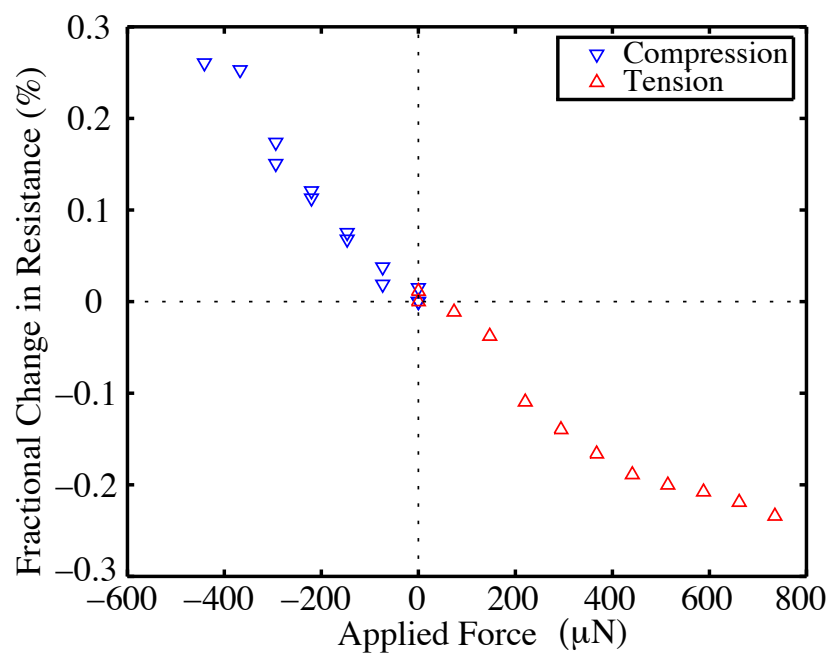

Fig. 7. Piezoresistance behavior of MUMPs-fabricated S-Curl device.

Figure 6 presents the data for the SUMMiT-fabricated U-Spring device and the MUMPs-fabricated Folded-Beam device. The results for both mechanisms reveal that the electrical resistance increased nonlinearly as the applied force increased. This non-linear increase in resistance was observed regardless of the force's direction. The fractional change in resistance for a beam in bending is small at low deflections and becomes more sensitive as deflection increases.

\subsection{Combined Loads}

The S-Curl and Snake devices were designed and tested to explore the effects of combined tension, compression and bending loads on piezoresistive behavior. Acquiring numerous data points at high applied forces was inhibited by the out-of-plane instability of the combined loading devices. Consequently, only data for relatively low applied forces are presented here.

The results of the S-Curl tests, shown in Fig. 7, illustrate a rise in resistance when the device was compressed. This increase in resistance became slightly steeper, i.e., more sensitive, at higher applied forces, much like the U-Spring devices. This behavior could be attributed to the combined or additive effect

of the compressive and bending stresses on the device, both of which induce an increase in resistance.

When the S-curl was put in tension and bending, its resistance initially decreased rapidly and then leveled off at higher applied forces. Initially the tensile stress in the S-curl dominated, resulting in a linear decrease in resistance. As the applied force continued to rise, the effect of bending stresses began to dominate, counteracting the decrease in resistance caused by the tensile stress. 


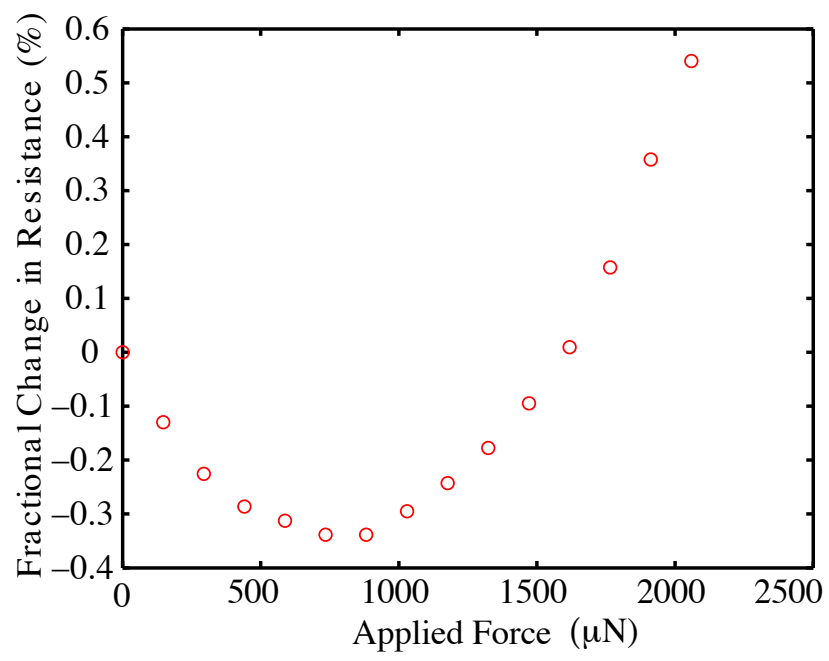

Fig. 8. Piezoresistive behavior for the Snake mechanism.

The piezoresistance effect in combined loads was further shown with the Snake mechanism. As Fig. 8 shows, the resistance of the Snake device initially decreased sharply, began to level off at approximately $800 \mu \mathrm{N}$, and then increased nonlinearly. Just as with the S-Curl device, the Snake device experienced both tension and bending loads. The trends seen in the data can be explained by the hypothesis that, when initially stretched, the dominant stress state was tension. This resulted in a decrease of resistance. As the deflection increased, causing higher stress levels, the bending stresses and resultant rise in resistance became dominant over the resistance drop due to tension.

\subsection{Summary of Results}

The experimental data show tensile stress induces a nearly linear decrease in resistance with a piezoresistive sensitivity similar to published empirical values for $n$-type polysilicon [31]. The piezoresistive sensitivity has been described in terms of a unitless piezoresistive gauge factor, $G$, calculated as

$$
G=\left(\frac{1}{\varepsilon}\right) \frac{\Delta R}{R}=\pi_{l} E+1+2 \nu
$$

for which $\varepsilon$ is the applied axial strain, $\pi_{l}$ is the longitudinal piezoresistance coefficient, $E$ is the modulus of elasticity, and $\nu$ is Poisson's ratio. As summarized in Table 4, the experimental gauge factors calculated from the tensile stress measurements of Fig. 5 show reasonable agreement with previous empirical data for polycrystalline silicon [31]. Since the gauge factor is expected to drop for highly-doped silicon, this agreement is entirely reasonable for MUMPsand SUMMiT-fabricated devices, which are both heavily doped. 
Table 4

Published and measured piezoresistance gauge factors for tensile stress, unitless. The published comparison data is reported in [31].

\begin{tabular}{ccccc} 
Published Values & SUMMiT & \multicolumn{3}{c}{ MUMPs } \\
& $3,4^{*}$ & $1^{*}$ & $2^{*}$ & $1,2^{*}$ \\
\hline-16 to -22 & -14.8 & -16.5 & -18.1 & -16.4 \\
\hline \multicolumn{3}{c}{${ }^{*}$ Polysilicon fabrication layer(s) }
\end{tabular}

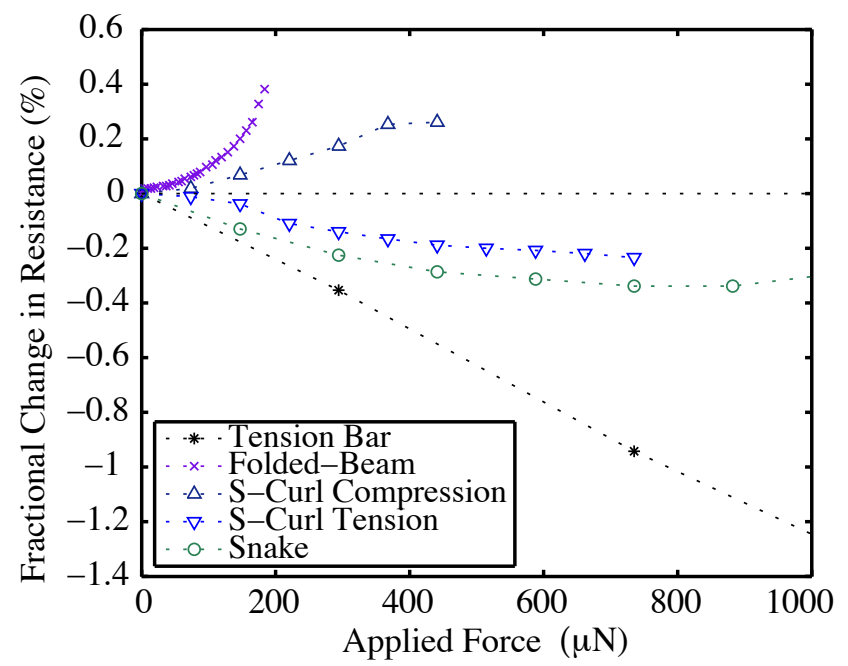

Fig. 9. Comparison of piezoresistive behavior in tension, bending and combined loads.

Figure 9 compares the resistance drop for tensile members with the nonlinear rise in resistance due to simple bending loads and the additive effect of combined loads on piezoresistive behavior. The figure's $x$-axis shows applied force to allow comparison in terms of a sample measurand for many applications. Although the piezoresistive sensitivity in bending is slightly inferior at lower force, the effect may be advantageous for piezoresistive devices which operate - and demand high piezoresistive sensitivity - at higher bending stresses or deflections. Furthermore, the bending and combined loading devices would be better suited for applications requiring significant displacement or deformation of the sensors during operation. Such deformation is not feasible with simple tensile members.

Figure 10 compares the resistance changes for the tensile and bending devices as a function of average stress magnitude. Note that, for tensile bars, stress is uniformly distributed, but bending stress varies throughout the beam. The average stress magnitude was found by averaging the absolute value of stress throughout the beam. The figure shows that the average stress magnitudes in each type of device are comparable. Hence, differences in stress magnitude are not responsible for the observed differences in resistance change trends. 


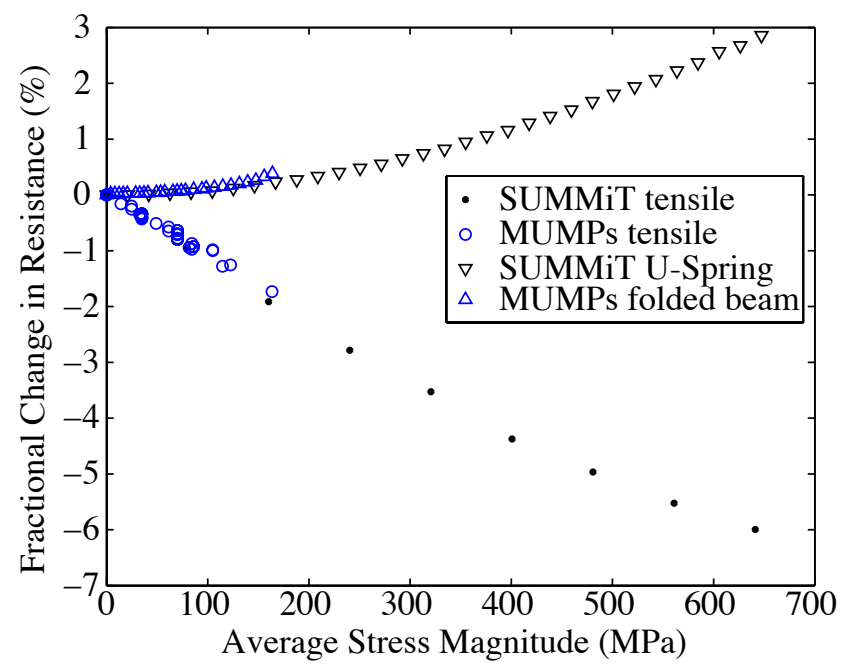

Fig. 10. Comparison of resistance change in tensile and bending devices vs. average stress

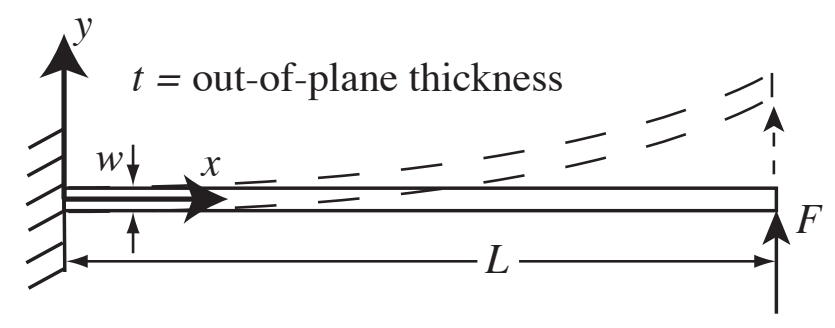

Fig. 11. End-loaded cantilever beam for resistance change model.

\section{Comparison to Linear Model}

\subsection{Modeling of the Piezoresistive Effect in Bending}

The previous section demonstrated that beams loaded in pure tension showed a linear change in resistance, as predicted by Eq. (3). However, the stress state is more complex for beams in bending. In order to make a comparison, this section develops a simple model of the resistance change for a beam in bending, assuming that Eq. (3) is satisfied. This simple model is based on the development in [25], pp.478-479.

Consider the cantilever beam loaded by a force $F$ at its end, shown in Fig. 11. By assuming that all current flows in the $x$-direction (or that $y$-direction current flows are negligible), we may approximate the resistance along this beam as

$$
R=\int_{0}^{L} \rho_{l}(x) \mathrm{d} x
$$


where $\rho_{l}(x)$ is the resistance per unit length, which is assumed to vary along the beam. We can approximate the conductance per unit length $g_{l}$ by integrating the conductance across the width $w$ of the beam, giving

$$
g_{l}=\frac{1}{\rho_{l}}=\int_{-w / 2}^{w / 2} \frac{t \mathrm{~d} y}{\rho_{e}(x, y)}
$$

where $t$ is the thickness of the beam (out of the plane shown in Fig. 11), and $\rho_{e}(x, y)$ is the electrical resistivity. As the stress will depend on both $x$ and $y$, $\rho_{e}$ also changes with both $x$ and $y$ according to Eq. (3). Hence,

$$
\rho_{e}(x, y)=\rho_{0}\left[1+\pi_{l} \sigma_{l}(x, y)\right]=\rho_{0}\left[1+\pi_{l} \frac{M(x) y}{I}\right]
$$

where $\rho_{0}$ is the unstressed electrical resistivity, $M(x)$ is the internal moment along the beam (given by $M(x)=-F(L-x)$ ), and $I$ is the second moment of area of the beam's cross-section, which is equal to $w^{3} t / 12$ for a rectangular cross section (assumed here). Substitution of Eq. (7) into Eq. (6) gives

$$
g_{l}=\frac{1}{\rho_{l}}=-\frac{t w}{2 \rho_{0} \beta(L-x)} \ln \left(\frac{1-\beta(L-x)}{1+\beta(L-x)}\right)
$$

where $\beta$ is given by

$$
\beta=\frac{6 \pi_{l} F}{w^{2} t}
$$

Finally, substituting Eq. (8) into Eq. (5) and making the variable substitution $u=L-x$ gives

$$
R=\frac{2 \rho_{0} \beta}{w t} \int_{L}^{0} u\left[\ln \left(\frac{1-\beta u}{1+\beta u}\right)\right]^{-1} \mathrm{~d} u
$$

This equation can be evaluated using numerical integration to find the beam's resistance as a function of applied force.

\subsection{Model Validation}

The simple model represented by Eq. (10) is based on several assumptions, such as the negligence of $y$-direction stresses or currents. To test these assumptions, the model was validated against a nonlinear commercial finite element 


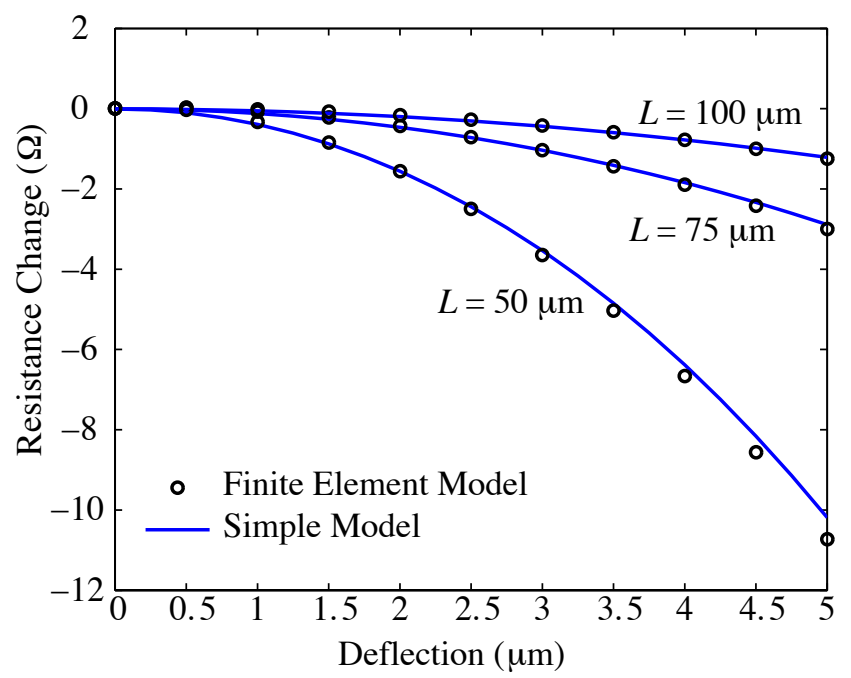

Fig. 12. Comparison of finite element simulations to predictions of the simple model for three cantilever beams.

code (ANSYS). The beam was modeled using 3-D solid226 elements, which model both the structural and piezoresistive behavior. The element allows the user to input $\pi$ coefficients from Smith's model directly into a matrix used in the algorithm. Therefore, the changes in resistivity in the three orthogonal directions and three cross directions from Eq. (1) were all represented. The piezoresistive coefficients used for validation, shown in Table 5, were approximated from the literature assuming a single-crystal silicon beam phosphorusdoped to $1 \times 10^{20} \mathrm{~cm}^{-3}$ [32]. For the simple model, $\pi_{l}$ was set equal to $\pi_{11}$ from Table 5 . Note that by comparing the full single-crystal silicon model with the simple model, we test the assumptions required to reduce Eq. (1) to Eq. (3) as well as the assumptions made in deriving Eq. (10).

For validation, three beams were modeled and compared. Each had a width of $3 \mu \mathrm{m}$ and a thickness of $3.5 \mu \mathrm{m}$. The lengths of the beams were 50, 75, and $100 \mu \mathrm{m}$. Figure 12 shows the predicted resistance change from each beam computed by the simple model and by ANSYS for a $5 \mu \mathrm{m}$ deflection of each beam. As the figure shows, the simple model agrees with the computationally intensive finite element model very well, demonstrating that the assumptions made in the simple model are appropriate.

Table 5

Piezoresistive $(\pi)$ coefficients used for FEM, from [32].

\begin{tabular}{ccc} 
Coeff. & Value & Units \\
\hline$\pi_{11}$ & $-39 \times 10^{-5}$ & $\mathrm{MPa}^{-1}$ \\
$\pi_{12}$ & $28 \times 10^{-5}$ & $\mathrm{MPa}^{-1}$ \\
$\pi_{44}$ & $-16 \times 10^{-5}$ & $\mathrm{MPa}^{-1}$ \\
\hline
\end{tabular}




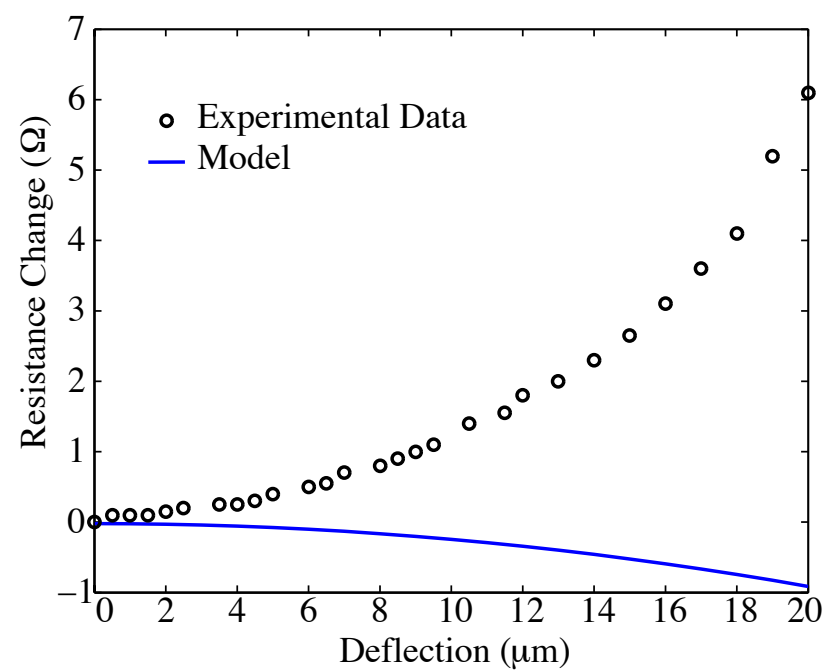

Fig. 13. Prediction of the simple model compared to experimental data for the folded-beam device.

\subsection{Comparison of Linear Model with Experimental Data}

After it was validated, the simple model was compared to experimental data for a beam in bending. Specifically, the folded-beam suspension shown in Figs. 2 and 3 was modeled by treating it as a total of 16 cantilever beams, each with half of the length of a single fixed-guided beam. Since the beams are arranged in both series and parallel with each other, the total device resistance is four times the resistance of a single cantilever, and the total device displacement is four times the displacement of a cantilever. Figure 13 shows the measured change in resistance compared to the change predicted by the simple model. For the simple model, $\pi_{l}$ was taken to be $-10.9 \times 10^{-5} \mathrm{MPa}^{-1}$, which is the value determined from the experimental tension data for the polysilicon layers used for the folded-beam device. The comparison demonstrates that the model predicts resistance change inaccurately, with both the magnitude and the trend (decreasing versus increasing resistance) of the model being incorrect. We conclude that the simple model, based on linearity of piezoresistance, is not sufficient to model the piezoresistance behavior of a device in bending. The next section examines this conclusion in more detail.

\subsection{Further Exploration of the Linear Model}

All of the data for bending of $n$-doped polysilicon beams show that the resistance rises with increasing deflection or force. However, the mathematical nature of the linear piezoresistive model precludes an upward trend in resistance for a flexure in bending. This inadequacy can be seen in a simple demonstration. Figure 14 shows a flexure being pressed downward by a force. 


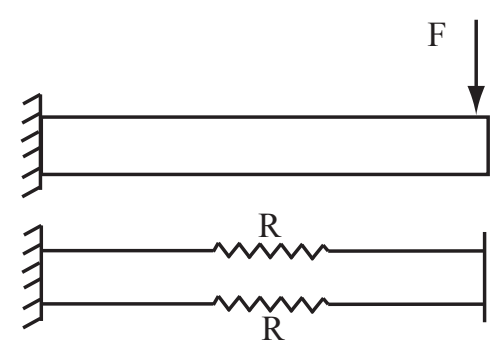

Fig. 14. Unstressed cantilever and resistor schematic.

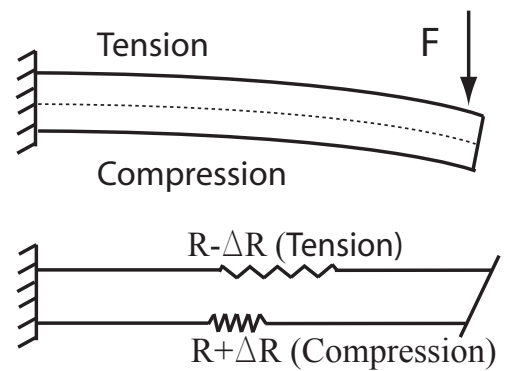

Fig. 15. Stressed Cantilever and resistor schematic.

In the figure, the beam is represented electrically by two resistors in parallel. The top resistor represents the part of the beam that will be placed in tension during bending; the bottom resistor represents material in compression. The unstressed resistance of the resistors in parallel $\left(R_{T}\right)$ is given by

$$
R_{T 0}=\left(\frac{1}{R}+\frac{1}{R}\right)^{-1}=\frac{R}{2}
$$

As the flexure is bent, the top half of the flexure is put in tension and the bottom half is put in compression, with the stress magnitudes in each half being the same. Representing the tension and compression in the flexure by the relative deformation of the resistors shown in Fig. 15, the linear piezoresistive model predicts that the resistance of the top half will drop by an amount $\Delta R$, while the resistance of the top half will rise by $\Delta R$. The total resistance is then given by

$$
R_{T}=\left(\frac{1}{R-\Delta R}+\frac{1}{R+\Delta R}\right)^{-1}=R_{T 0}-\frac{\Delta R^{2}}{2 R}
$$

Hence, the model predicts that the total resistance will always drop for any bending deformation, contrary to the experimental data.

As evident from these experimental results, the linear piezoresistance model failed to capture the trend and the magnitude of the piezoresistive effect for the simple bending device. In fact, regardless of the sign or magnitude of 
the piezoresistance coefficients used in the analyses, the model predicts a decrease in resistance due to bending stresses, while every bending test shows an increase in resistance. Consequently, a more complete nonlinear model of piezoresistance is needed to predict the effects of bending and combined loads on piezoresistance, Such a model could be based on previously-published data demonstrating small nonlinearities in the piezoresistive effect in silicon [33]. Such a model would facilitate the design, optimization and implementation of integral piezoresistive sensors and may provide greater insights into the physical phenomenon of piezoresistivity.

\section{Conclusion}

This paper described an experimental investigation of the piezoresistive effect of polysilicon in bending and combined loads. The design and experimental setup of several $n$-type polysilicon test structures was provided, as well as the results from experimentation. Tensile or compressive stresses cause a linear change in resistance, but bending stresses induce a nonlinear rise in resistance for $n$-type polysilicon. A combination of tension, compression and bending stresses add together, with the tensile and compressive stresses dominating at lower applied force levels. The experimental results demonstrate the failure of linear piezoresistance models to predict the piezoresistive effect of polysilicon in bending. This represents a motivation for the development of a more complete nonlinear model of piezoresistance which accurately predicts piezoresistance in bending. Such a model may shed greater light on the physical phe-

nomenon of piezoresistance and will facilitate the design and optimization of integral piezoresistive sensors.

\section{Acknowledgements}

The authors gratefully acknowledge the support of the National Science Foundation through NSF grant CMS-0428532. The Integrated Microfabrication Lab at BYU also provided equipment and expertise for this work.

\section{References}

[1] C. S. Smith, Piezoresistance effect in germanium and silicon, Physical Review 94 (1954) 42-49. 
[2] Y. Sun, B. J. Nelson, MEMS for cellular force measurements and molecular detection, International Journal of Information Acquisition 1 (2004) 23-32.

[3] P. French, Polysilicon: a versatile material for microsystems, Sensors and Actuators A: 99 (2002) 3-12.

[4] W. P. Eaton, J. H. Smith, Micromachined pressure sensors: review and recent developments, Smart Materials and Structures. 6 (1997) 530-539.

[5] N. Maluf, An Introduction to Microelectromechanical Systems Engineering, Artech House Publishers, Boston, USA, 1994.

[6] J. W. Gardner, Microsensors: Principles and Applications, John Wiley and Sons, USA, 1994.

[7] S. Sze (Ed.), Semiconductor Sensors, John Wiley and Sons, New York, New York, 1994.

[8] P. Ciureanu, S. Middelhoeck, Thin Film Resistive Sensors, Institute of Physics Publishing, New York, 1992.

[9] R. K. Messenger, T. W. McLain, L. L. Howell, Feedback control of a thermomechanical inplane microactuator using piezoresistive displacement sensing, American Society of Mechanical Engineers, Dynamic Systems and Control Division (Publication) DSC 73 (2004) 1301-1310.

[10] P. B. Ruffin, MEMS-based sensor arrays for military applications, Proceedings of SPIE - The International Society for Optical Engineering 4700 (2002) 111 121.

[11] V. Gridchin, V. Lubimsky, Phenomenological model of the piezoresistive effect in polysilicon films, Russian Microelectronics 32 (2003) 205-213.

[12] W. P. Eaton, Surface micromachined pressure sensors, Ph.D. thesis, The University of New Mexico, Albuquerque, New Mexico (1997).

[13] P. Kleimann, B. Semmache, M. L. Berre, D. Barbier, Stress-dependent hole effective masses and piezoresistive properties of p-type monocrystalline and polycrystalline silicon, Physical Review B 57 (1998) 8966-8971.

[14] S. Durand, C. Tellier, Linear and non-linear piezoresistance coefficients in cubic semiconductors., J. Phys. III France 6 (1996) 237-266.

[15] P. French, A. Evans, Piezoresistance in polysilicon and its applications to strain gauges, Solid-State Electronics 32 (1989) 1-10.

[16] P. French, A. Evans, Polycrystalline silicon strain sensors, Sensors and Actuators A: Physical 8 (1985) 219-225.

[17] M. M. Mandurah, The physical and electrical properties of polycrystallinesilicon, Ph.D. thesis, Stanford University (1981).

[18] R. W. Keyes, The effects of elastic deformation on the electrical conductivity of semiconductors, Solid state physics 11 (1960) 149-218. 
[19] C. Herring, E. Vogt, Transport and deformation-potential theory for manyvalley semiconductors with anisotropic scattering, Physical Review 101 (1956) 944-961.

[20] J. S. Shor, L. Bemis, A. D. Kurtz, Characterization of monolithic n-type 6HSIC piezoresistive sensing elements, IEEE Transactions on Electron Devices 4 (1994) 661-665.

[21] M. E. Fauver, D. L. Dunaway, D. H. Lillenfeld, H. G. Craighead, P. G. H, Microfabricated cantilevers for measurement of subcellular and molecular forces, IEEE Trans. Biomed. Eng. 45 (1998) 891-898.

[22] J. K. Anderson, L. L. Howell, J. W. Wittwer, T. W. McLain, Piezoresistive sensing of bistable micro mechanism state, Journal of Micromechanics and Microengineering 16 (5) (2006) 943-950.

[23] J. F. Nye, Physical Properties of Crystals, Oxford University Press, Oxford, UK, 1985.

[24] D. W. Burns, Micromechanical integrated sensors and the planar processed pressure transducer, Ph.D. thesis, University of Wisconsin, Madison, WI (1988).

[25] S. D. Senturia, Microsystem Design, Kluwer Academic Publishers, Boston, USA, 2001.

[26] B. Bahreyni, C. Shafai, Application of twin-beam structures for estimation of material properties and sensor fabrication, Can. J. Elect. Comput. Eng. 31 (2) (2006) 85-88.

[27] D. A. Koester, R. Mahadevan, B. Hardy, K. Markus, MUMPs Design Handbook, Rev. 5.0, Cronos Integrated Microsystems, 2000.

URL http://www. memsrus.com.

[28] J. J. Sniegowski, Multi-level polysilicon surface-micromachining technology: applications and issues, in: Proc. of the ASME Aerospace Division, 1996 International Mechanical Engineering Congress and Exposition, Vol. AD-Vol. 52, 1996, pp. 751-759.

[29] J. W. Wittwer, T. Gomm, L. L. Howell, Surface micromachined force gauges: uncertainty and reliability, Journal of Micromechanics and Microengineering 12 (2002) 13-20.

[30] L. L. Howell, Compliant Mechanisms, John Wiley and Sons, USA, 2002.

[31] P. French, A. Evans, Polycrystalline silicon as a strain gauge material, J. Phys. E: Sci. Instrum. 19 (1986) 1055-1058.

[32] O. Tufte, E. Stelzer, Piezoresistive properties of silicon diffused layers, Journal of Applied Physics 34 (1963) 313-318.

[33] K. Matsuda, K. Suzuki, K. Yamamura, Y. Kanda, Nonlinear piezoresistance effect in silicon, Journal of Applied Physics 73 (1993) 1838-1847. 